\title{
A BLENDED DEEP LEARNING APPROACH FOR OUTDOOR SCENE IMAGE ENHANCEMENT
}

\author{
Deepa Abin \\ Research Scholar and Assistant Professor, Computer Department, Pimpri Chinchwad College of \\ Engineering, Akurdi, \\ Pune, Maharashtra, India \\ deepa.abin@pccoepune.org \\ Sudeep D.Thepade \\ Professor, Computer Department, Pimpri Chinchwad College of Engineering, Akurdi, \\ Pune, Maharashtra, India \\ sudeep.thepade@pccoepune.org \\ Aditya Yadav \\ Student, Computer Department, Pimpri Chinchwad College of Engineering, Akurdi, \\ Pune, Maharashtra, India \\ adityayadav5oct@gmail.com \\ Prathamesh Bhagat \\ Student, Computer Department, Pimpri Chinchwad College of Engineering, Akurdi, \\ Pune, Maharashtra, India \\ bprathamesh41@gmail.com
}

\begin{abstract}
:
The most significant issue with visibility in outdoor images is atmospheric haze and inadequate lighting. If the haze is dense and the lighting is uneven, the problem becomes more difficult, resulting in poor image contrast. This paper provides an integrated method using Contrast Limited Adaptive Histogram Equalization(CLAHE) and AOD-net machine learning algorithm to reduce haze and enhance contrast of image. As images are pre-processed with CLAHE, a lightweight model efficiently dehazes the image. Experiments are conducted utilizing a variety of hazy pictures obtained from RESIDE- $\beta$ dataset, and performance of proposed method was checked by PSNR, SSIM, PIQE, NIQE, BRISQUE, Entropy metrics. Results reveal that the suggested technique can successfully restore the contrast of haze affected pictures by dehazing them.
\end{abstract}

Keywords: Computer Vision; Image processing; CLAHE; Convolutional Neural Network; Single Image dehazing.

\section{Introduction}

For many computer vision applications, poor visibility in inclement weather is a key issue. The majority of autonomous surveillance, intelligent cars, outdoor object identification, and other systems presume that the input images are clear. Unfortunately, this isn't always the case in many scenarios, thus increasing visibility is an unavoidable duty. The presence of haze significantly decreases the clarity of outdoor photograph taken in bad weather, many computer vision applications including detection and recognition, face the problem of haze in environment. Haze is caused when sunlight collides with microscopic pollution particles in the air, particles absorb certain amount of light and rest is scattered. The image taken in an outdoor scene is also severely damaged due to contrast change from original irradiance at the moment of capture [Nishino et al. (2012)]. This is caused mostly by the presence of haze, smoke, and fog[Narasimhan and Nayar(2002)]. Such raw images need to be processed and dehazed to extract more details from it. Image dehazing techniques can be classified as single image dehazing methods and multi-image dehazing methods. Several input photographs are acquired under varied air circumstances and processed to remove the haze in the multi-image dehazing approach; the outcomes of this method are extremely appealing. The multi-image dehazing's primary flaw is in the acquisition stage. We need to capture many input pictures for processing in this phase, therefore this form of dehazing requires a lot of memory, 
a lot of time, and is extremely tough to complete. On the other hand, single picture dehazing takes only one hazy image as input and processes it to remove haze. Lately, few more techniques of dehazing image are introduced such as contrast-based and statistical techniques. In contrast-based technique, the haze is eradicated by increasing contrast of hazy image whereas, statistical technique leverages the image's depth information to remove haze. In this article, the proposed approach, improves the contrast of image as well as dehazes the image by first applying CLAHE on hazy input image, then processes it with AOD net machine learning algorithm, which is a Convolutional Neural Network (CNN). The main contributions of the paper include:

We have proposed our own extended convolutional neural network, as per the specific needs to correct the over enhanced CLAHE processed image. We have used the method of AOD-Net[Li et al. (2017)] to unify two variables namely transmission matrix and atmospheric model into one variable and estimate it to recover the clean image. We further dehaze the image by further considering the over enhancement of CLAHE as haze.

This paper is structured into several sections. Section 2 presents related work and background study, proposed method and experimental setup is also elaborated in Section 2. Section 3 shows results explained in three parts Subjective, Objective and Graphical respectively . Finally, in section 4 Conclusion and the Future scope of proposed method is given.

\section{Related Work}

In [Soni and Mathur(2020)], Badal et al. proposed a method which fuses CLAHE and Guided filter mechanism. First CLAHE was used on the input hazy image, then guided filter mechanism was used to overcome drawbacks due to CLAHE, prior to this white balancing was also used on input hazy image before applying proposed method to make image look more natural. Results revealed that the method was quite effective but low contrast images could not be processed well. Ansia et al. [Ansia and Aswathy(2015)], suggested a single image dehazing technique which primarily uses saliency map of white balanced image along with morphological opening and CLAHE for haze removal. The method is simple and does not have complex calculations which result in less time complexity.

In [Liu et al. (2019)], Zheng et al. calculated the unknown parameters in atmospheric scattering model, arguing the real relationship between the original and hazy pictures can be rather complicated and the atmospheric scattering model may not entirely depict it. They proposed a significant method based on machine learning algorithm $(\mathrm{CNN})$. The algorithm restores clear images without adhering to atmospheric scattering model. It helped to overcome common drawbacks of other methods like excessive edge sharpening and color darkening.

In [Qin et al. (2019)], Xu et al. proposed another single image dehazing technique based on the fact that haze is distributed unevenly in image therefore different weights should be given to thin and thick haze region pixels. When compared to previous state-of-the-art approaches, the suggested network considerably aids in the restoration of colour accuracy and picture details.

In [Ancuti et al. (2010)], Codruta et al. introduced a single image dehazing technique based on multi scale image fusion. From the given hazy input image, two images are generated using Contrast Enhancement and White balancing. To avoid loss of details while performing Contrast Enhancement proper weights are applied. In further processing Laplacian pyramid and Gaussian pyramid is calculated and all levels are merged, adhering bottom up approach.

Blind Haze Separation[Shwartz et al. (2006)] states outdoor images, proposes less visibility due to attenuation, scattering under hazy conditions. A serious issue is that variable spatial minimization of contrast by the airlight that is scattered by the atmospheric fog particles, which are received by the camera. By providing a depth map for an image, computer vision algorithms have recently demonstrated that pictures contain disruptions such as haze. The most important aspect is that such a subtraction functions as a scene recovery of ambient light. It is obtained by the recognition of a polarization-filtered picture. The recovery necessitates airlight specifics. Previously, these details were obtained by calculating pixels in sky photos in prior investigations. The proposed technique obtains an approach for the details that are necessary for the separation of airlight from computations, which are recovered in the absence of vision, then the recovery of contrast, without user's interaction, and also without the reality of sky inside frame.

In [Mei et al. (2019)], Mei et al. seeked to find non-linear transformation between input hazy image and its corresponding ground-truth. They suggested a method which can restore $4 \mathrm{k}$ image at once without breaking it into parts. Their method is based on U-Net like architecture. One of the most often used dehazing techniques is contrast enhancement.

In [Zhang et al. (2017)], Zhang et al. proposed a model based on assumption that attenuation and scattering is homogeneous for each channel. The method estimates the airlight for every pixel, eliminates it from processing image, then removes the haze by calculating the transmission and intensity of changing illumination. One drawback of this method is in the areas of grasses and leaves, where there is some color wrapping.

In most dehazing algorithms boundaries of image was not preserved properly. To resolve this Yibo[Tan and Guoyu Wang(2020)], et al. introduced a new approach based on Markov random field(MRF) and superpixels. 
The proposed approach also improves the contrast of image. MRF helps to prevent block effect on the edges, thus preserving boundary effectively.

Many approaches for improving image contrast have been presented, which can be classified into direct methods and indirect methods. Because of its simplicity and explicitness, histogram equalization approaches have been widely used among indirect methods. The basic idea behind HE is to make the enhanced image's histogram resemble a uniform distribution so that the image's dynamic range can be fully used [Kotkar and Gharde(2013)]. HE increases the image contrast globally by adjusting the image's globally dispersed intensities to improve contrast. The more advanced version of HE is CLAHE, which divides the given image into smaller segments and Histogram equalization is applied to each segment, also in this method a maximum value known as clip limit is used to clip the histogram and redistribute it in respective color space. CLAHE limits the amplification of noise in the image so that image appears more natural [Dhariwal (2011)]. The dark channel prior (DCP)[He et al. (2011)] is a sort of statistics in which specific pixels in at least one of the RGB channels have an extremely low intensity. The intensity of these black pixels in that specific channel is primarily due to air light in hazy photos. As a consequence, these black pixels may be used to accurately measure haze transmission in the picture. It recovers a clear, haze-free picture and generates a reasonable depth map. users' interaction, as well as the absence of a realistic sky within the frame

Zhang et al.[Zhang et al. (2015)], proposed a haze model for differing light sources, halos. It includes transmission map, airlight and halos. The input is an halo image. Then optimization problem divides it into halo and halo-free images. Halo-free images required advance processing. Airlight and transmission map estimation is major contribution. This is a simple and economical method. However haze-free results are not upto mark compared to other methods.

Manjunath et al.[Manjunath and Phatate(2016)], proposed an elementary but potent prior which provides adaptation of detailed algorithm for single image dehazing. This algorithm works on multiple scattering technique so input image provided is blurrier. This method employs a multiple scattering mechanism, resulting in a blurrier input picture. Dehazing becomes very straightforward and efficient when this approach is used with a single picture dehazing model. This method, which is based on local content, is more sensitive than color and may be used on a wider range of pictures. Many physical models are used to address this disadvantage.

The AIPNet[Wang et al. (2019)] shows the statistical analysis that the luminance channel of $\mathrm{YCbCr}$ is affected the most by haze, hence visual contrast of image is enhanced by identifying haze using luminance channel. CSIDNet[Sharma et al. (2020)] uses dark channel prior, illumination channel prior and three convolutional layers to obtain dehazed images. MSE as a loss function is used for training, based on MSE values for varied number of layers and parameters, the adequate parameters and layers were used. The model is light weight and obtains visually clear images by not excessive dehazing in sky regions of light and fine haze. MSCNN[Ren et al. (2016)] creates a coarse-scale transmission matrix, which is then refined. For training the network, a method is proposed to synthesize hazy images using depth dataset. The coarse scale network learnt the comprehensive features of transmission map, the fine scale network learnt the local features of transmission map. Thus, the fine scale network enhances the roughly produced transmission map of coarse scale network.

For the hazy picture formation process, the atmospheric scattering model is described as

$$
\mathrm{I}(y)=\mathrm{J}(y) \mathrm{t}(y)+\mathrm{B}(1-\mathrm{t}(y))
$$

Where I (y) is the observed hazy image and $\mathbf{J}(\mathrm{y})$ is the scene radiance to be recovered. There are two crucial parameters to be estimated: ' $\mathrm{B}$ ' signifies the total amount of light in the atmosphere where as 't(y)' (transmission matrix) defined as:

$$
\mathrm{t}(y)=\mathrm{e}^{-\beta \mathrm{d}(\mathrm{y})}
$$

where $\beta$ is the scattering coefficient of the atmosphere, $d(y)$ being the separation between the camera and the object. Most of the other CNN models recover the transmission matrix followed by estimating global atmospheric light; a haze-free image is then recovered using the atmospheric scattering model. As the transmission matrix and the atmospheric light are estimated individually, the errors compound and magnify one another. AOD-Net[Li et al. (2017)] unifies the two parameters transmission matrix $(\mathrm{t}(\mathrm{y}))$ and atmospheric light $(\mathrm{B})$ into one formula, i.e., $\mathrm{K}$ (y).

Where,

$$
\begin{aligned}
& J(y)=\frac{1}{t(y)} I(y)-B \frac{1}{t(y)}+B . \\
& J(y)=K(y) * I(y)-K(y)+b
\end{aligned}
$$

$$
K(y)=\frac{\frac{1}{t(y)}(I(y)-B)+(A-b)}{I(y)-1}
$$

It directly reduces the picture pixel domain reconstruction errors.. $\mathrm{K}$ is estimated using $\mathrm{CNN}$ followed by clean image generation by substituting $\mathrm{K}$ in above equation Eq. (4). 


\begin{tabular}{|c|c|c|c|c|}
\hline Ref & $\begin{array}{l}\text { Method } \\
\text { Name }\end{array}$ & Dataset & Pre-Processing & Methodology \\
\hline $\begin{array}{l}\text { [Li et al. } \\
(2017)]\end{array}$ & AOD-Net & $\begin{array}{l}\text { Synthesize hazy images } \\
\text { using ground truth and } \\
\text { NYU2 Depth database, } \\
\text { setting different } \\
\text { atmospheric lights. }\end{array}$ & None & $\begin{array}{l}\text { Unifies two parameters, } \\
\text { transmission matrix and } \\
\text { atmospheric light to one } \\
\text { parameter which is estimated by } \\
\text { network. }\end{array}$ \\
\hline $\begin{array}{l}\text { [Sharma et } \\
\text { al. (2020)] }\end{array}$ & CSID-Net & RESIDE & $\begin{array}{l}\text { Dark channel prior, } \\
\text { illumination channel } \\
\text { prior. }\end{array}$ & $\begin{array}{l}\text { Uses only three convolution } \\
\text { layers to obtain haze free image. }\end{array}$ \\
\hline \begin{tabular}{|l} 
[Wang et \\
al. (2019)]
\end{tabular} & AIP-Net & $\begin{array}{l}\text { IMAGENET } \\
\text { ILSVRC2012, } \\
\text { Middlebury Stereo } \\
\text { datasets, NYU-DEPTH } \\
\text { DATASET. }\end{array}$ & None & $\begin{array}{l}\text { Luminance channel is regarded } \\
\text { as a hazy channel. AIPnet } \\
\text { provides Y' which is combined } \\
\text { with } \mathrm{Cr}, \mathrm{Cb} \text { of original channels } \\
\text { to produce dehazed image. } \\
\text { Feature extraction, multi scale } \\
\text { restoration. }\end{array}$ \\
\hline $\begin{array}{l}\text { [Ren et al. } \\
(2016)]\end{array}$ & MSCNN & $\begin{array}{l}\text { Synthesize hazy images } \\
\text { using ground truth and } \\
\text { NYU2 Depth database, } \\
\text { estimating atmospheric } \\
\text { lights. }\end{array}$ & None & $\begin{array}{l}\text { Coarse-scale network, its fine- } \\
\text { scale network to generate a } \\
\text { refined transmission map, } \\
\text { estimate atmospheric light to } \\
\text { dehaze image. }\end{array}$ \\
\hline $\begin{array}{l}\text { [Qin et al. } \\
(2019)]\end{array}$ & FFA-Net & $\begin{array}{l}\text { Reside, NYU Depth V2, } \\
\text { Middlebury Stereo } \\
\text { datasets. }\end{array}$ & None & $\begin{array}{l}\text { Consists of block architecture } \\
\text { with local residual learning, and } \\
\text { Feature Attention module which } \\
\text { comprises of channel wise } \\
\text { attention and pixel wise } \\
\text { attention. }\end{array}$ \\
\hline $\begin{array}{l}\text { [Mei et al. } \\
(2019)]\end{array}$ & PFF-Net & $\begin{array}{l}\text { RESIDE, NTIRE2018 } \\
\text { Image Dehazing Dataset. }\end{array}$ & None & $\begin{array}{l}\text { It consists of three modules: } \\
\text { encoder, feature transformation } \\
\text { and decoder. }\end{array}$ \\
\hline $\begin{array}{l}\text { [Liu et al. } \\
(2019)]\end{array}$ & GMAN & RESIDE & None & $\begin{array}{l}\text { Encoder-decoder structure with } \\
\text { down- and up-sampling factor of } \\
\text { 2, residual learning, VGG16 } \\
\text { transfer learning. }\end{array}$ \\
\hline
\end{tabular}

Table 1. Comparison analysis of existing methods

\section{Proposed Method}

The proposed method first enhances the local contrast of image using CLAHE followed by CNN algorithm AODNet for clean image generation, with better contrast even in dark conditions. 


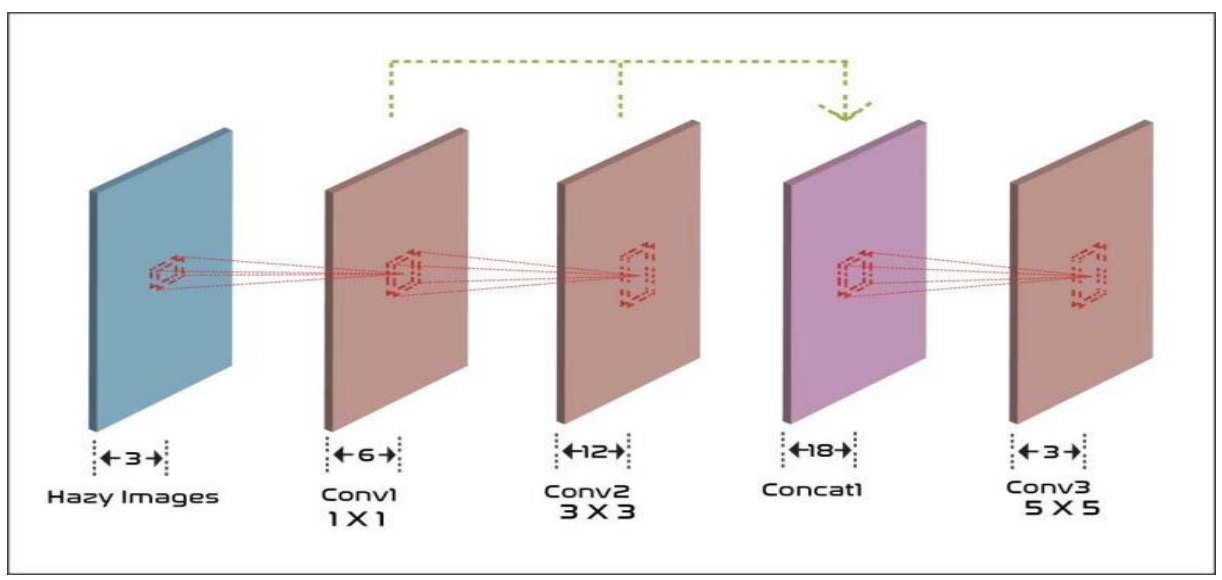

Fig 1. Convolution Layers

Preactivation: CLAHE is applied on each of the three red, green, blue channels separately with a tile grid of $8 \times 8$ and clip limit 2.0 with OpenCV. Similarly, CLAHE is also applied on Luminance(Y) channel of YCbCr and experimented against the same network. The 3 channels are further passed to the network. The Figure 1, depicts the suggested model's convolution layers. It depicts the Kernel size for each convolutional layer in aXb format. The figures in between the arrows show the output channel for the respective layers.

The network has 3 convolutional layers and a concatenation layer. First convolutional layer is fed with 3 RGB CLAHE processed channels with Kernel size of 1 , stride of 1 and no padding being applied. This layer produces 6 channels of feature maps. These channels after passing through the relu layer are input to the second convolutional layer with Kernel size of 5, stride of 1 and padding 2. This layer produces 12 channels. The concatenation layer further concatenates 6 and 12 relu processed channels of first and second layers respectively to produce output of 18 channels. These channels are convoluted with Kernel size 3, stride 1, padding 1 to produce a 3-channel output layer.

Dehazed image: This network estimates $\mathrm{k}$ as output of the final layer. Clean image is recovered by substituting $\mathrm{k}$ in Eq. (4). The parameters are set in comparison with clear image provided along with hazy image.

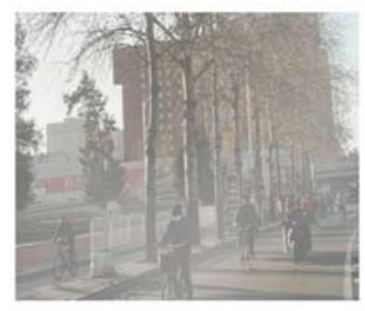

Input Hazy Image

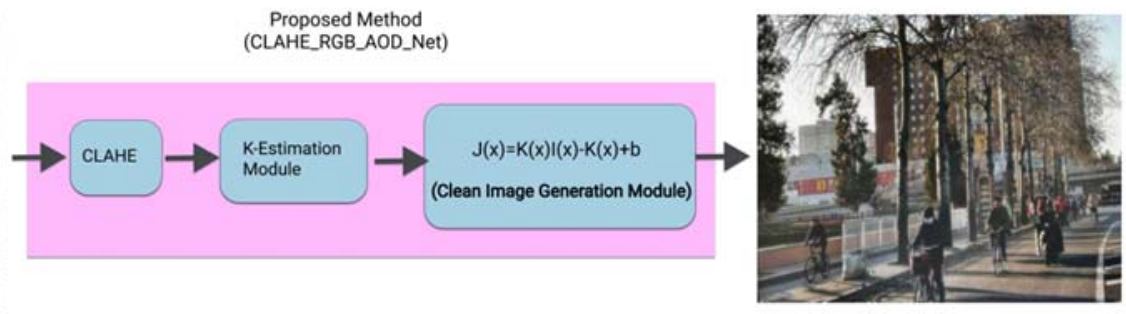

Output Image

Fig 2. Flow diagram of proposed method.

Necessity of CLAHE with machine learning model: Although CLAHE enhances contrast locally in a fixed tile(grid) size, the contrast of objects is sometimes unequally enhanced around different edges, also object of interest may be over enhanced due to background conditions. The noise in the images also amplifies with CLAHE. Most of the machine learning networks aim to train the model using the images by considering a hazy image associated with clear image. The model being trained, sets the parameters based on different hazy images for corresponding to single clear image, thus the model fails to set parameters complying actual contrast and there by fine details of objects. As proposed method first uses CLAHE on images, the contrast of hazy images is enhanced, these may over enhance contrast of objects and also amplify the noise, the machine learning network further learns to enhance image adequately, reduce the noise and thereby dehazes the image. 


\section{Experimental Setup}

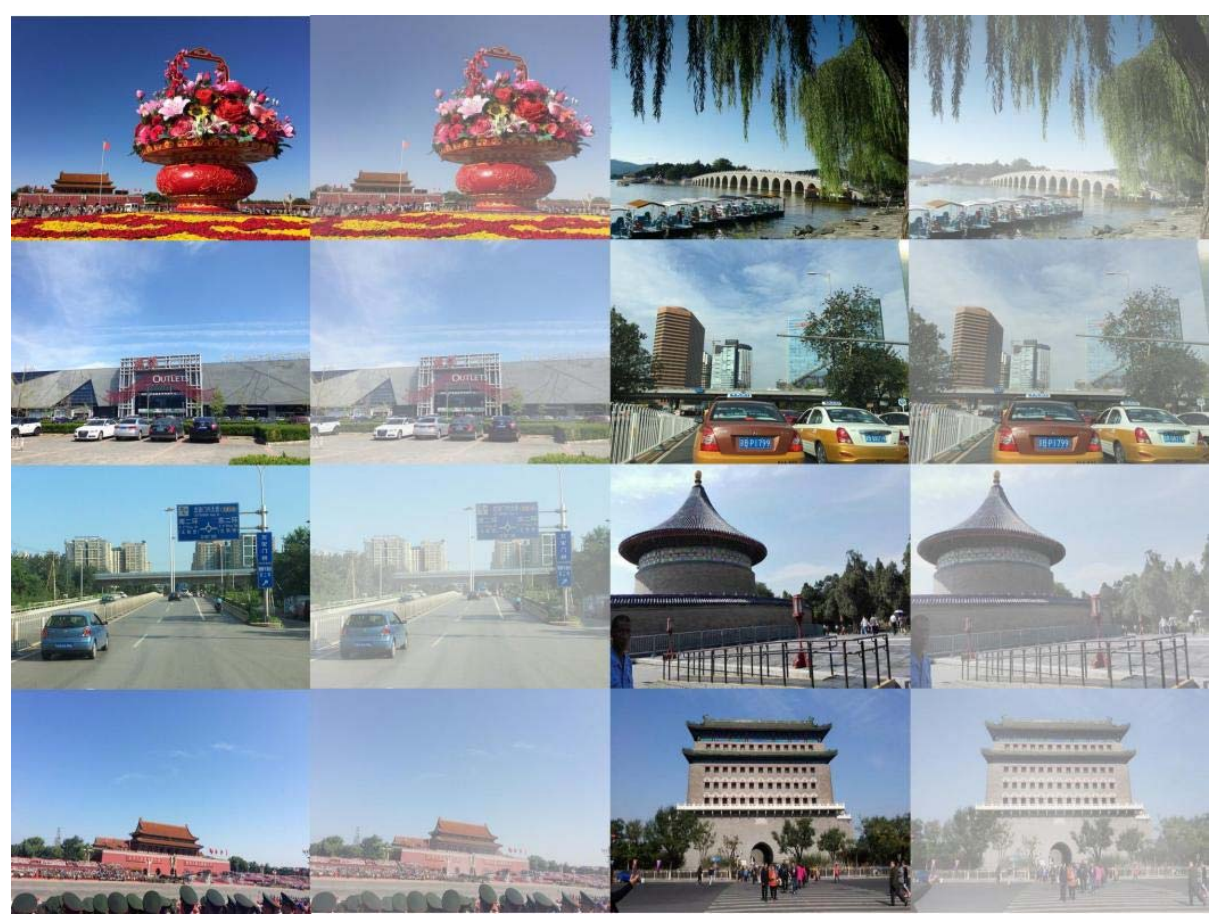

Fig 3. Sample of images from RESIDE- $\beta$ dataset

The RESIDE- $\beta$ [Li et al. (2019)] dataset consists of 2061 clear images, with each clear image (ground truth) corresponding set of 35 synthetic hazy images in Outdoor Training Set (OTS) is available. The training of proposed model was done on 18,200 images selected from OTS with their respective ground truths (original clear image). The model was then tested against 3,640 hazy images. The hazy images are first split into RGB channels and CLAHE is applied on each channel, further they are merged and passed to network. The network is further trained on just 11 epochs to produce the results. Original AODNet[Li et al. (2017)] model was also trained for comparison. FFANet[Qin et al. (2019)], PFFNet[Mei et al. (2019)], GMAN[Liu et al. (2019)] methods were trained and experimented using same dataset as the proposed method. Experimentation using CLAHE on Y channel of $\mathrm{YCbCr}$, was also carried out as pre-processing for network. The SSIM, PSNR metric were computed and is shown in Figure 7. 
The model was also assessed using no reference image quality assessment based on PIQE, NIQE, BRISQUE and Entropy metrics were computed and plotted for comparison on 5180 images for each method. The approach was to test the model with CLAHE on different color channels for the same neural network model.
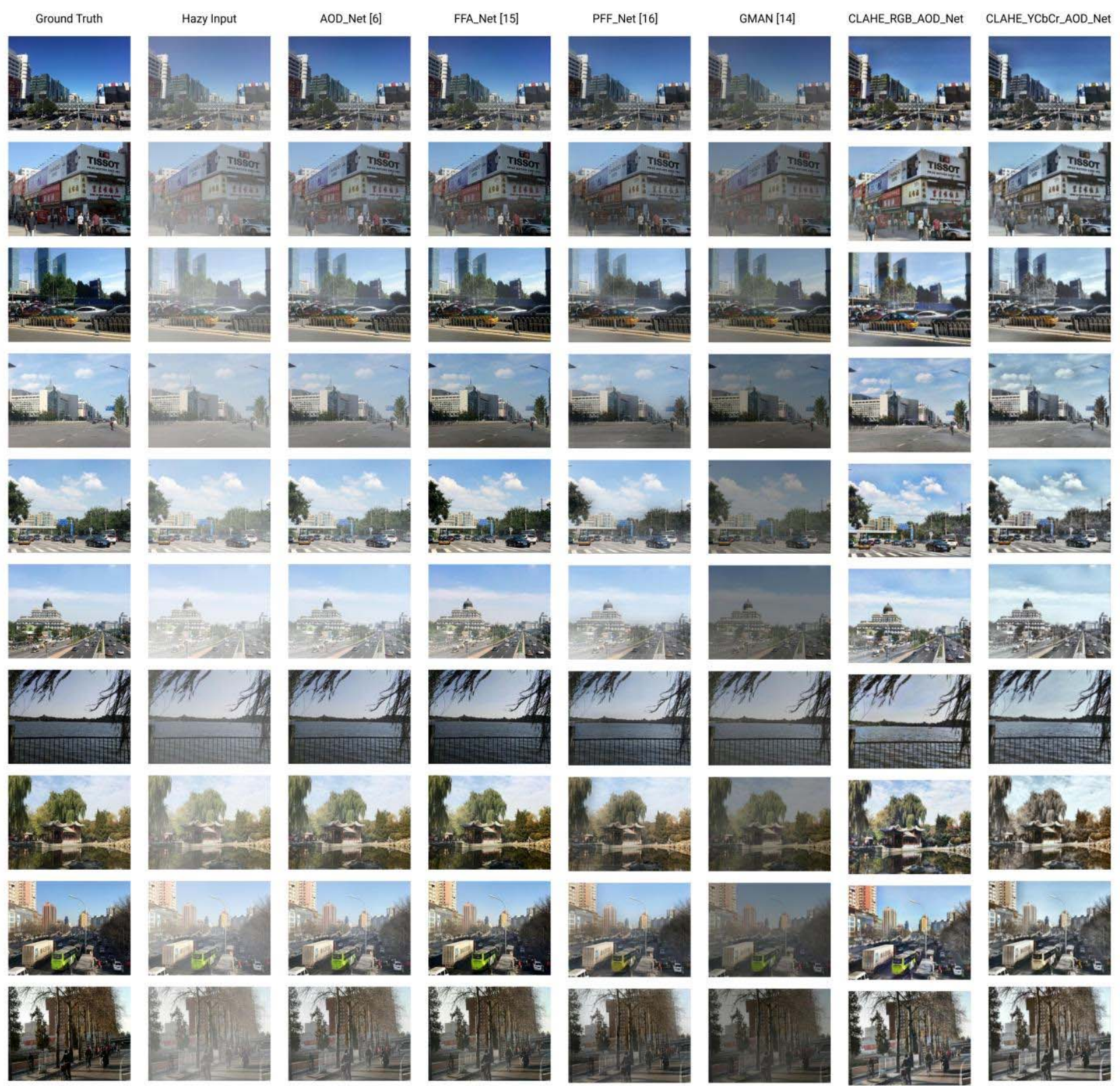

Fig 4. Comparison analysis of different methods on RESIDE- $\beta$ dataset images

\section{Results and Discussion}

This section consists of subjective, objective, graphical results and discussions. The notation AOD Net refers to pure AOD_Net[Li et al. (2017)], whereas CLAHE_RGB_AOD_Net and CLAHE_YCbCr_AOD_Net are the proposed methods where we have used our architecture for CLA $\bar{H} E$ processing, the term AŌD in our methods only refers to the idea of using one parameter of AOD_Net[Li et al. (2017)] for dehazing image.

\subsection{Subjective results}

Proposed method shows clear image subjectively as compared to other networks. As the method enhances CLAHE processed images, the noise produced is suppressed considerably by network, also the image has adequate contrast enhancement for objects to be processed further. As it primarily tries to reduce noise amplified by CLAHE, the PSNR metrics of proposed method is slightly lesser in most cases then AODnet as shown in fig (7), however it is sufficient enough for optimal object detection. 
Ground Truth
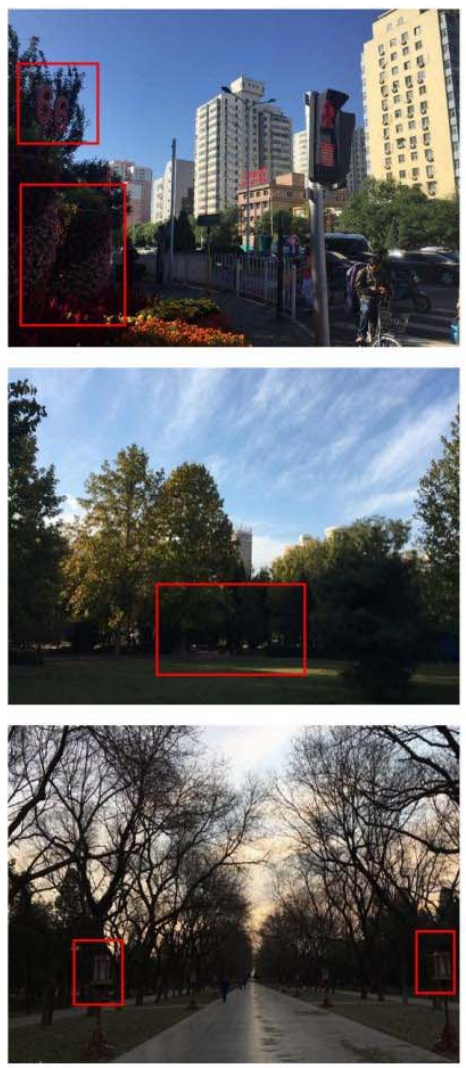

Hazy Input
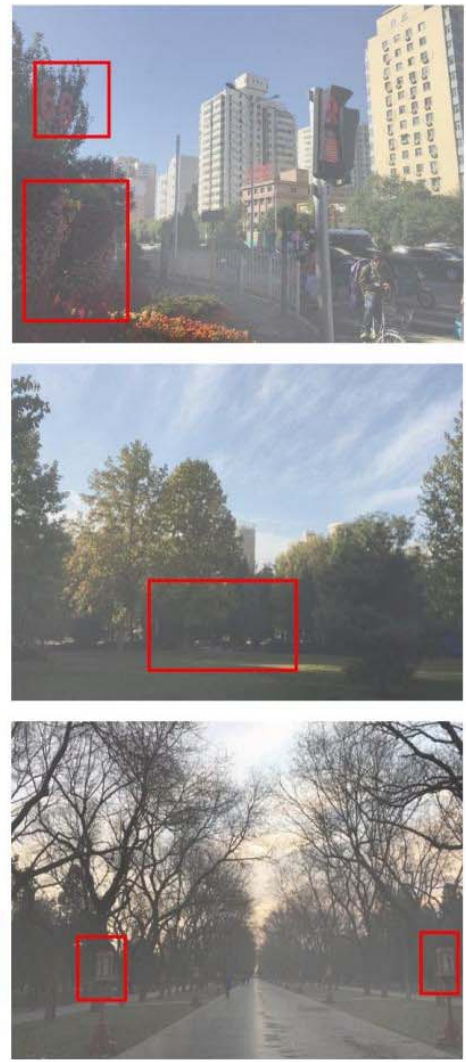

CLAHE_RGB_AOD_Net
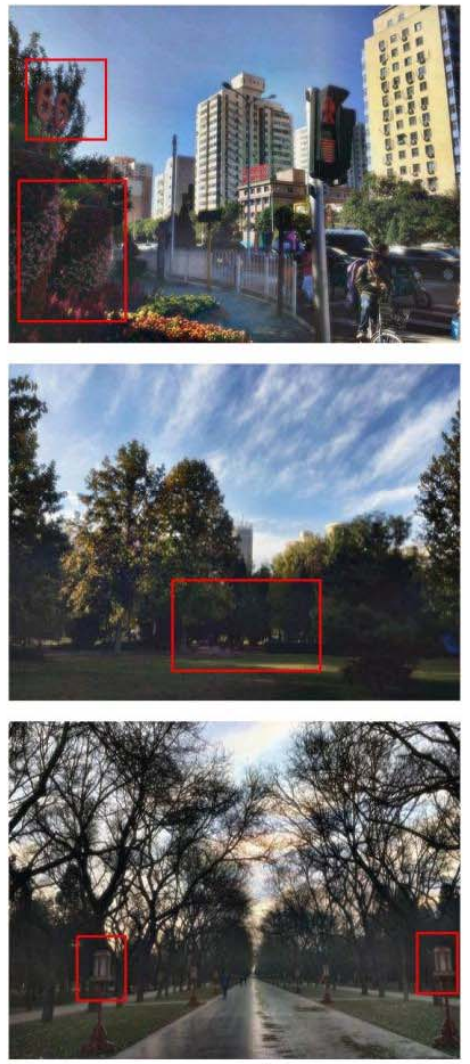

Fig 5. Details enhanced by proposed method.

From Figure 5, it can be clearly observed, that proposed method not only helps to dehaze image, but also enhances quality of image. In first row of Figure 5, although the flowers are not clearly visible in ground truth image, after applying CLAHE_RGB_AOD_Net the flowers are clearly visible despite giving hazy input image to the algorithm.

Similarly in second row of Figure 5, it can be noticed that the region beneath the tree is quite enhanced by the algorithm despite feeding Hazy input image to algorithm. Similarly, in third row of Figure 5, the street lamps that were obscured in ground truth image are easily noticeable after applying CLAHE_RGB_AOD_Net algorithm.

From this, it can be clearly stated that unlike other algorithm that focuses only on $\bar{d}$ ehazing the image, the proposed method also enhances the details of image.

\subsection{Objective results}

PSNR ratio is used to compare the quality of the original and reconstructed images. The better the quality of the compressed or reconstructed image, the higher the PSNR. The Structural Similarity Index (SSIM) is a scale that evaluates the degradation of image quality due to processing such as data compression or transmission losses. PSNR, SSIM is based on observable image structures. SSIM analyses the original and processed images to determine the differences. It does not say which of the two is superior, but it does indicate how big of a perceptual difference there is between the two by assigning a value between 0 and 1 . The greater the SSIM number, the better is processed image. 


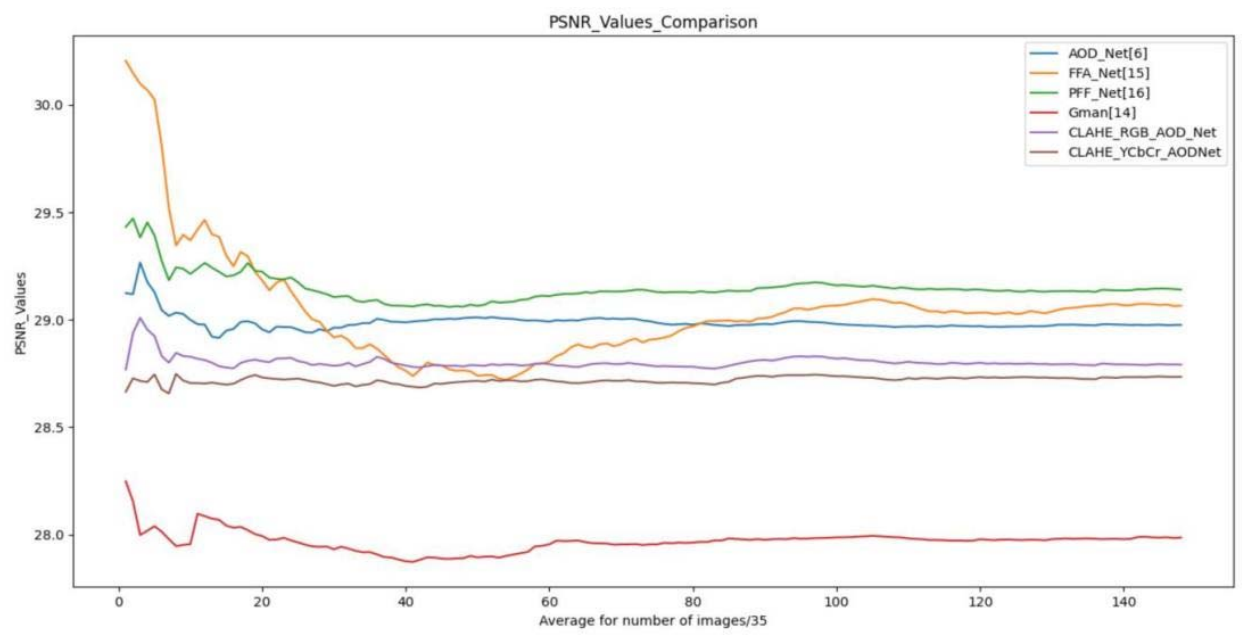

Fig 6. Performance analysis using PSNR on different methods

Figure 6, depicts comparison of PSNR metric for images evaluated by different methods, the result per index is cumulative of earlier indices. PSNR and SSIM metric is higher most of the times for FFA_Net. As illustrated in Figure 7, the proposed method shows consistency for different types of hazy images being compared.

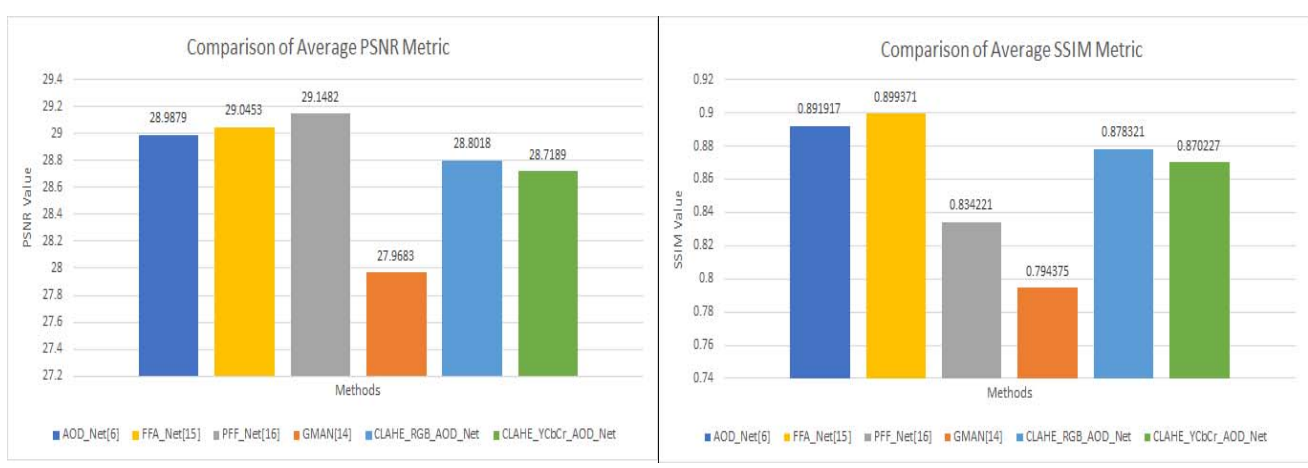

Fig 7. Comparison of SSIM and PSNR Metric of various methods

Figure 7, illustrates the quantitative results of average PSNR and SSIM metrics for set of 35 images per index for six different techniques experimented. It can be observed here, that though PSNR and SSIM metric for proposed approach is moderate, but it is very much less computationally intensive.

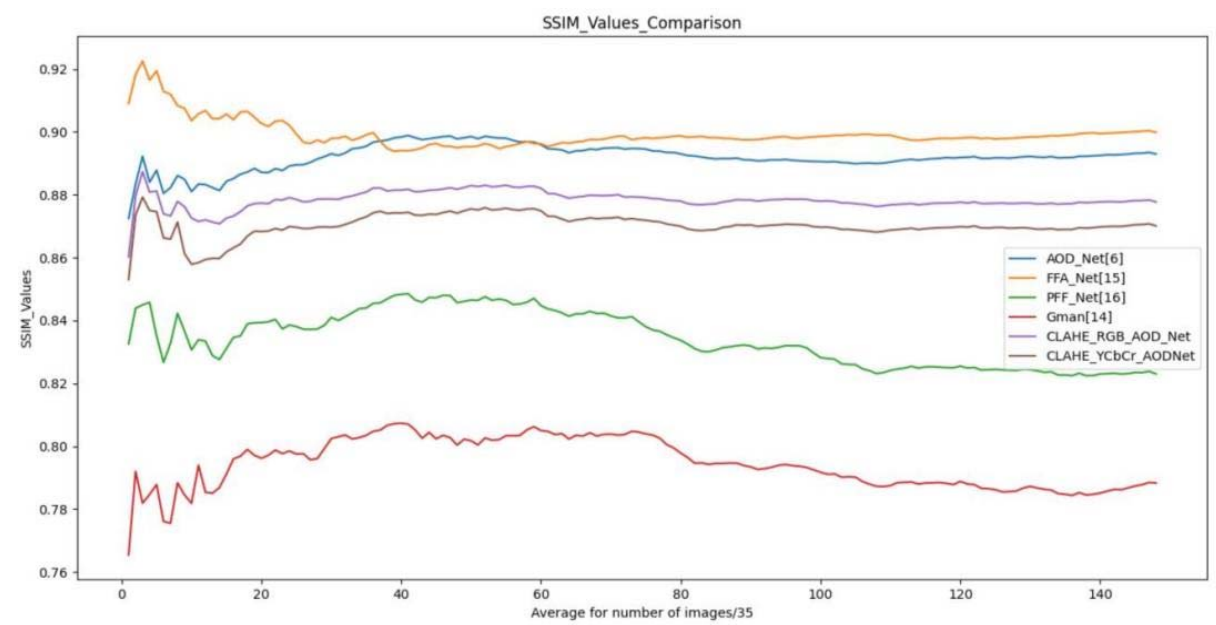

Fig 8. Performance analysis using SSIM on different methods 
Figure 8, shows comparison of SSIM with different methods. As observed, the proposed method has higher SSIM metric most of the times. This justifies the claim of images being structurally similar and more adequate for object defining tasks. In above results, CLAHE_RGB_AOD_Net is the technique in which CLAHE is applied on RGB channels and the image is dehazed with proposed network. Similarly, CLAHE_YCbCr_AOD_Net is the technique where CLAHE is applied only on luminance channel and the image produced is further dehazed with the network. AOD_Net label indicates the results for original AOD_Net[Li et al. (2017)] architecture. Similarly, FFA_Net, PFF_Net, Gman label indicate results for FFANet, PFFNet and Gman respectively. All the techniques are experimented on same set of hazy images.

\subsection{Graphical results}

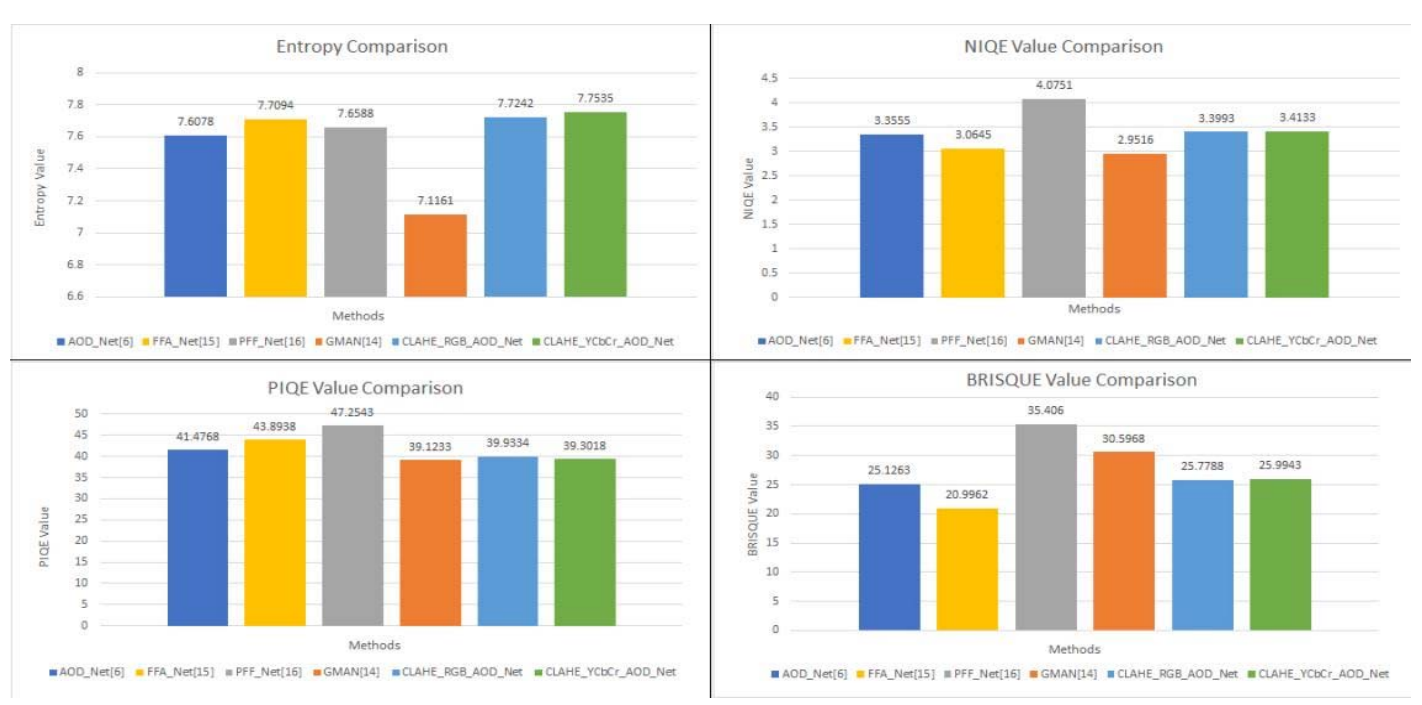

Fig 9. Entropy, NIQE, PIQE, BRISQUE value performance comparison of different methods

Here, Entropy of proposed method in RGB and $\mathrm{YCbCr}$ is better than the individual existing models. A low score of the Naturalness Image Quality Evaluator (NIQE) [Mittal et al. (2013)], Perception based Image Quality Evaluator (PIQE) [Venkatanath et al. (2015)] and Blind Image Spatial Quality Evaluator (BRISQUE) [Mittal et al. (2012)] suggests a higher level of perceptual quality. CLAHE_RGB_AOD_Net has shown better NIQE and PIQE respectively. Though BRISQUE score is not at par relatively, but the subjective and objective evaluation if considered together, the proposed approach has demonstrated better results.

\subsection{Discussions}

Lately, few techniques for dehazing image are introduced such as contrast-based and statistical techniques. Some statistical techniques use atmospheric model with depth information to retrieve transmission matrix and there by generate clean images, whereas some techniques directly train over input hazy images to generate clear images. These architectures though dehaze the image, are heavy to train on large custom datasets for large epochs. Also, for real time applications it is better to use a light architecture so as to dehaze image with less computational power quickly. Understanding the requirements, we propose a light weight model. CLAHE alone dehazes the images, but over enhances the image. Thus, we control the over enhancement of image with network. AODNet[Li et al. (2017)] states the haze removal was not much efficient with just 5 convolutional layers, they had to use 3 concatenations more for multiscale design, to get the adequate results. We have used 3 convolutional layers and a concatenation layer to produce the adequate results. Our algorithm also enhances the contrast over image due to CLAHE, thus the produced output image is subjectively more promising, to be optimally used for computer vision applications. As we are first applying CLAHE, some uniform large regions like sky have some noise even after passing through network. We conducted experimentation on different color spaces with CLAHE but couldn't remove all noise in the large uniform regions. CLAHE on YCbCr worked identical to CLAHE on RGB before passing to network.

\section{Conclusion}

The suggested method not only beats different state-of-the-art dehazing models, but it also creates a standard, a compact model having minimal resource requirements. The improved pictures acquired using the suggested technique successfully preserve the speed-accuracy trade-off. The suggested network produces impressive results, indicating its use in a variety of critical and real-time applications. The SSIM value clearly shows the technique is far better and can be very useful in object detection algorithms. With 11 epochs, this model was trained on 
18,200 pictures. Model was also trained on 3640 pictures as dataset, the results were similar to the proposed network with some degradation in image quality. Minor drawback of suggested technique is that the model increases noise in sky areas conveyed by CLAHE less often, so work needs to be done further to reduce noise in sky areas. CLAHE was also applied on YCbCr color space with CLAHE on Y channel but CLAHE on RGB channels produced better results. Subjectively the results are more visually enhanced than the clear image referred for experimentation, making it more adequate for object detection modules.

\section{Acknowledgements}

We thank the research community and our well wishers.

\section{References}

[1] B. Soni and P. Mathur (2020), "An Improved Image Dehazing Technique using CLAHE and Guided Filter," 2020 7th International Conference on Signal Processing and Integrated Networks (SPIN), pp. 902- 907, doi: 10.1109/SPIN48934.2020.9071296

[2] Narasimhan, S.G., Nayar, S.K. (2002),Vision and the Atmosphere. International Journal of Computer Vision 48, $233-254$. https://doi.org/10.1023/A:1016328200723

[3] Nishino, K., Kratz, L. \& Lombardi, S. Bayesian Defogging (2012). Int J Comput Vis 98, 263-278 . https://doi.org/10.1007/s11263-0110508-1

[4] Matlin, Erik \& Milanfar, Peyman. (2012). Removal of Haze and Noise from a Single Image. SPIE Conference on Computational Imaging. SPIE Proceedings, vol. 8296. 8296. 17-. 10.1117/12.906773.

[5] Kotkar, V.A., \& Gharde, S.S. (2013). REVIEW OF VARIOUS IMAGE CONTRAST ENHANCEMENTTECHNIQUES. International Journal of Innovative Research in Science, Engineering and Technology, 2, 2786-2793.

[6] B. Li, X. Peng, Z. Wang, J. Xu and D. Feng (2017), "AOD-Net: All-in-One Dehazing Network," IEEE International Conference on Computer Vision (ICCV), 2017, pp. 4780-4788, doi: 10.1109/ICCV.2017.511.

[7] Sharma, T., Agrawal, I. \& Verma, N.K. (2020), CSIDNet: Compact single image dehazing network for outdoor scene enhancement. Multimed Tools Appl 79, 30769-30784. https://doi.org/10.1007/s11042- 020-09496-z

[8] Ren W., Liu S., Zhang H., Pan J., Cao X., Yang MH. (2016) Single Image Dehazing via Multi-scale Convolutional Neural Networks. In: Leibe B., Matas J., Sebe N., Welling M. (eds) Computer Vision - ECCV 2016. ECCV 2016. Lecture Notes in Computer Science, vol 9906. Springer, Cham. https://doi.org/10.1007/978-3-319-46475-6_10

[9] Dhariwal, S. (2011). Comparative Analysis of Various Image Enhancement Techniques

[10] Ansia, S. \& Aswathy, A.L. (2015). Single Image Haze Removal Using White Balancing and Saliency Map. Procedia Computer Science. 46. 10.1016/j.procs.2015.01.042.

[11] Kotkar, V.A., \& Gharde, S.S. (2013). REVIEW OF VARIOUS IMAGE CONTRAST ENHANCEMENTTECHNIQUES. International Journal of Innovative Research in Science, Engineering and Technology, 2, 2786-2793.

[12] K. He, J. Sun and X. Tang (2011), "Single Image Haze Removal Using Dark Channel Prior," in IEEE Transactions on Pattern Analysis and Machine Intelligence, vol. 33, no. 12, pp. 2341-2353, doi: 10.1109/TPAMI.2010.168.

[13] A. Wang, W. Wang, J. Liu and N. Gu (2019), "AIPNet: Image-to-Image Single Image Dehazing with Atmospheric Illumination Prior," in IEEE Transactions on Image Processing, vol. 28, no. 1, pp. 381-393, doi: 10.1109/TIP.2018.2868567.

[14] Z. Liu, B. Xiao, M. Alrabeiah, K. Wang and J. Chen (2019), "Single Image Dehazing with a Generic Model-Agnostic Convolutional Neural Network," in IEEE Signal Processing Letters, vol. 26, no. 6, pp. 833- 837, doi: 10.1109/LSP.2019.2910403.

[15] Qin, Xu \& Wang, Zhilin \& Bai, Yuanchao \& Xie, Xiaodong \& Jia, Huizhu. (2019). FFA-Net: Feature Fusion Attention Network for Single Image Dehazing.

[16] Mei K., Jiang A., Li J., Wang M. (2019) Progressive Feature Fusion Network for Realistic Image Dehazing. In: Jawahar C., Li H., Mori G., Schindler K. (eds) Computer Vision - ACCV 2018. ACCV 2018. Lecture Notes in Computer Science, vol 11361. Springer, Cham. https://doi.org/10.1007/978-3-030-20887-5_13.

[17] N. Venkatanath, D. Praneeth, Bh. M. Chandrasekhar, S. S. Channappayya, and S. S. Medasani (2015). "Blind Image Quality Evaluation Using Perception Based Features", In Proceedings of the 21st National Conference on Communications (NCC). Piscataway, NJ: IEEE.

[18] Mittal A., A. K. Moorthy, and A. C. Bovik (2012). "No-Reference Image Quality Assessment in the Spatial Domain." IEEE Transactions on Image Processing. Vol. 21, Number 12, pp. 4695-4708.

[19] Mittal, A., R. Soundararajan, and A. C. Bovik (2013). "Making a Completely Blind Image Quality Analyzer." IEEE Signal Processing Letters. Vol. 22, Number 3, pp. 209-212.

[20] Codruta Orniana Ancuti, Cosmin Ancuti and Philippe Bekaert (2010), "Effective Single Image Dehazing by Fusion," IEEE 17th International Conference on Image Processing. pp. 3541-3544.

[21] Zhang, Y. Cao, S. Fang, Y. Kang, and C. W. Chen (2017), 'Fast haze removal for nighttime image using the reflectance prior', In 2017 IEEE Conference on Computer Vision and Pattern Recognition.

[22] Yibo Tan, Guoyu Wang (2020). "Image haze removal based on superpixels and markov random field." Transactions on IEEE Access, 8(4):60728-60736.

[23] J. Zhang, Y. Cao, and Z. Wang (2015), 'Nighttime Haze Removal with Glow and Multiple Light Colors', In 2015 IEEE International Conference on Computer Vision.

[24] Manjunath.V, Revanasiddappa Phatate (2016), “A Single Image Haze Removal Algorithm Using Color Attenuation Prior”, International Journal of Scientific and Research Publications, Issue 6, pages 291-297

[25] Sarit Shwartz, Einav Namer and Yoav Y. Schechner (2006), "Blind Haze Separation,” IEEE Computer Society Conf. Computer Vision and Pattern Recognition (CVPR'06), vol. 2, pp. 1984-1991.

[26] B. Li et al. (2019), "Benchmarking Single-Image Dehazing and Beyond," in IEEE Transactions on Image Processing, vol. 28, no. 1, pp. 492-505, doi: 10.1109/TIP.2018.2867951. 


\section{Authors Profile}

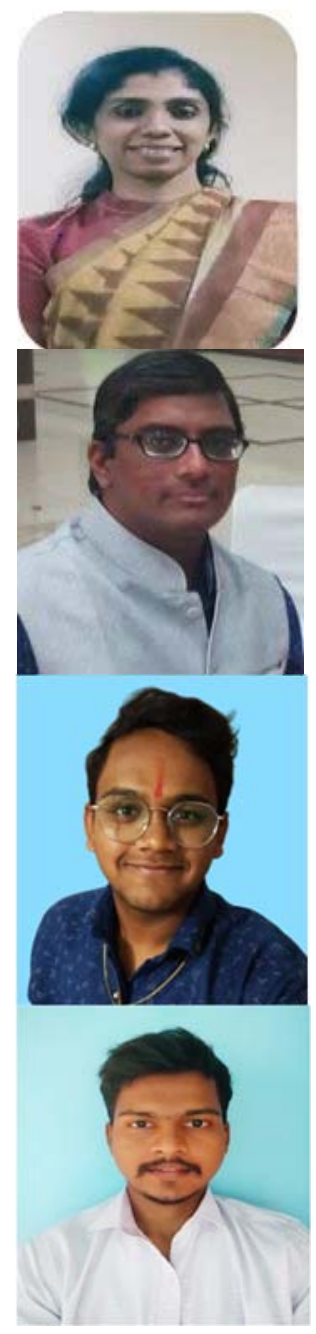

Deepa Abin is currently Assistant Professor in Computer Engineering Department at Pimpri Chinchwad College of Engineering affiliated to

Savitribai Phule Pune University, Pune, Maharashtra, India. She is currently pursuing Ph.D. in Computer Engineering from PCCoE-SPPU. She has published several papers in International, National Conferences and Journals. Her domain of interest is Video Enhancement, Image Processing, Machine Learning and Artificial Intelligence.

Sudeep D. Thepade is Professor with Pimpri Chinchwad College of Engineering, Pune, India. He has more than 320 research articles in Journals and Conferences to his credit. $\mathrm{He}$ is reviewer for several International Journals including IEEE and IET. He is member of international bodies like IAENG, IACSIT, ICGST. He is member Board of studies, Research Review Committee at several Universities. He is guiding few Ph.D. Scholars and has guided many Masters and Bachelors projects.

Aditya Yadav is currently an undergraduate student pursuing Bachelor of Engineering at Pimpri Chinchwad College of Engineering affiliated to Savitribai Phule Pune University, Pune, Maharashtra, India. He is currently working as technical team member at Team Automatons, Robotics Team of PCCoE-SPPU.

Prathamesh Bhagat is currently an undergraduate student pursuing Bachelor of Engineering at Pimpri Chinchwad College of Engineering affiliated to Savitribai Phule Pune University, Pune, Maharashtra, India. 